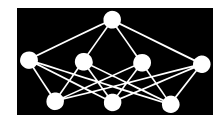

\title{
HYBRID NEURAL NETWORK BASED RAINFALL PREDICTION SUPPORTED BY FLOWER POLLINATION ALGORITHM
}

\author{
S. Chatterjee, B. Datta ${ }^{\dagger}$ N. Dey
}

\begin{abstract}
The present work proposes a hybrid neural network based model for rainfall prediction in the Southern part of the state West Bengal of India. The hybrid model is a multistep method. Initially, the data is clustered into a reasonable number of clusters by applying fuzzy c-means algorithm, then for every cluster a separate Neural Network $(\mathrm{NN})$ is trained with the data points of that cluster using well known metaheuristic Flower Pollination Algorithm (FPA). In addition, as a preprocessing phase a feature selection phase is included. Greedy forward selection algorithm is employed to find the most suitable set of features for predicting rainfall. To establish the ingenuity of the proposed hybrid prediction model (Hybrid Neural Network or HNN) has been compared with two well-known models namely multilayer perceptron feed-forward network (MLP-FFN) using different performance metrics. The data set for simulating the model is collected from Dumdum meteorological station (West Bengal, India), recorded with in the 1989 to 1995. The simulation results have revealed that the proposed model is significantly better than traditional methods in predicting rainfall.
\end{abstract}

Key words: Artificial Neural Network, Flower Pollination algorithm, Rainfall Prediction, back propagation, gradient descent, fuzzy c-means

Received: November 1, 2016

DOI: $10.14311 / \mathrm{NNW} .2018 .28 .027$

Revised and accepted: November 1, 2018

\section{Introduction}

Rainfall is probably one of the most influential natural events and it highly affects the weather. As a result it plays a key role in the natural life and habitat of a demographic region. Rainfall quantity is a key factor in agriculture. Both less and excessive rainfall might reduce the agricultural production. Besides, excessive amount of rainfall causes natural disasters which put millions of lives at severe risk. The south-eastern part of Asia is highly dependent on annual rainfall. However, due to the affects mentioned earlier, it is imperative to accurately predict the

\footnotetext{
*Sankhadeep Chatterjee - Corresponding author; Department of Computer Science and Engineering, University of Calcutta, Kolkata, India, E-mail: sankha3531@gmail.com

${ }^{\dagger}$ Bimal Datta; Department of Computer Science and Engineering, Budge Budge Institute of Technology, Kolkata, India, E-mail: bkd_hetc@yahoo.co.in

${ }^{\ddagger}$ Nilanjan Dey; Department of Information Technology, Techno India College of Technology, Kolkata, India, E-mail: neelanjan.dey@gmail.com
} 
rainfall quantity. From the point of view of weather prediction, several attempts have been made by meteorologists to accurately predict the quantity of rainfall in a geographic region. Studies have revealed that the quantity of rainfall depends on other measurable weather parameters. However, such models involve computationally infeasible mathematical calculations in order to predict the rainfall quantity. Therefore, even after being accurate in several cases, such models are not suitable for rainfall predictions as it does not meet the current trend of predicting rainfall for small geographic regions [34]. A second type of rainfall prediction model has been proposed which utilizes machine learning models which aim at approximating the complex relation between the rainfall quantity and measurable weather parameters [15]. In case of real life applications, the machine learning based approach is highly suitable as it provides stable results in reasonable amount of time. Recent advancement in the research of weather predictions have indicated that Artificial Neural Networks (ANNs or NNs) could be a suitable choice for predicting different weather parameters $[4,10,11,14,28,29,38]$. Further, studies have established that the NN based models are robust, accurate and prone to noisy data which is common in weather prediction models [3,8,12,19,31,37], [16]. Rainfall prediction has attracted the researchers and several successful models have been proposed. Quantitative rainfall prediction in the Indian subcontinent using several NN based models have been proposed [33]. Nanda et al. [33] compared four different machine learning based models namely multilayer perceptron feed-forward network, ARIMA, Legendre polynomial equations, and FLANN models. The study revealed that FLANN based rainfall prediction model is most accurate. Hourly data has been utilized to predict short time rainfall prediction in Thailand [23]. The study utilized different weather parameters such as humidity, pressure, wet bulb temperature and cloudiness of sky to predict rainfall one to three hour ahead. The study has reported that wet bulb temperature is one of the key factors for such short term predictions using feature selection method.

Literature survey has revealed that ANN could be efficiently used for weather prediction tasks [26]. In spite of immense success of ANN based models in predicting weather parameters, it suffers from a significant problem regarding the training algorithms. The traditionally used training algorithms are based on gradient descent method. These algorithms try to find the optimal weight values of ANN by minimizing an error function which works as an estimator of how well an ANN is. However, these algorithms are local optimization algorithms which are ill suited for training ANNs. These algorithms do not ensure optimal weights for the ANN. Besides, recent studies have revealed that the performance of ANN is better than traditional classifiers in several engineering and science applications $[1,5,35]$.

Consequently, the present article proposes a Hybrid Neural Network (HNN) based machine learning model for rainfall prediction [39], [20], [9] based on weather data consisting of features namely vapor content, relative humidity, atmospheric pressure, and temperature. The data is gathered by Dumdum meteorological center situated at West Bengal, India within a span of six years. In the training phase the HNN model involves two different phases. In the first phase, fuzzy c-means clustering algorithm is used to cluster the dataset into a reasonable number of clusters. After that the data of each cluster is used to train a separate classifier. To overcome the problems faced in traditional ANNs, a recently proposed well 
known metaheuristic Flower Pollination algorithm (FPA) has been used to train the NNs in the HNN model. The prediction model is used for predicting rainfall quantity and also for a classification task where the data samples are considered to be in two different classes, one is 'Rain' which indicate the rainfall quantity on that day is positive. The second class is 'No Rain' which corresponds to data samples where the rainfall quantity is recorded ' 0 '. The proposed model has been compared with two different well-known classifiers namely MLP-FFN and NN trained with backpropagation algorithm by using different performance metrics namely accuracy, precision, recall and F-measure. The simulated experimental results revealed that the proposed model is superior to other models in predicting rainfall. Furthermore, to establish the ingenuity of the results achieved by the proposed model Wilcoxon Rank test with $5 \%$ level of significance is reported.

The rest of the article is arranged as follows; Section 2 introduced the proposed Hybrid Neural Network based model with details, including the multi-step training phase. Thereafter, Section 3 discussed experimental methods. Finally Section 4 reported the simulated results with an comparison with other classifier, and statistical significance test as well.

\section{Proposed method}

The current study proposes a novel hybrid neural network (or HNN) model for rainfall prediction. The training phase of the HNN method consists of two different stages. The first stage clusters the data points into a suitable number of clusters. Next, for each cluster a separate ANN model is used. Studies have revealed that traditional ANNs might not perform well if trained using gradient descent based algorithms [11], [10]. Thus, in the current study a well-known metaheuristic algorithm called the Flower pollination Algorithm is used to train the NNs. The following subsections discusses on the methods.

\subsection{Metaheuristic supported Neural Network}

Artificial Neural Networks or Neural Networks are widely used in prediction and classification tasks. It is achieved by training the NNs using a suitable amount of data regarding the pattern of our interest. Traditionally, gradient descent based algorithms are used to train NNs [30]. The quality of the trained network can be determined by measuring the deviation between the expected output and calculated output. The training algorithms are designed to minimize such an error function which estimates the quality of the NNs. In this process the weights of NNs are adjusted in such a way that the error function can be minimized as far as possible [6]. However, gradient descent based algorithms are ill suited to find the optimal weight values for the NNs. These algorithms are local search based algorithms and often stuck into local optima values in spite of the presence of a global optima value of the error function [36]. The problem is further escalated from the fact that the starting point of the searching process highly affects the probability of achieving the global optima. The problem can effectively solved using global optimization techniques in the training phase of NNs. Recent studies have revealed the potential of using metaheuristic algorithms in order to train NNs. 
A typical multilayer feed-forward network [13] consists of three types of neuron layers. First, in the input layer the number of neurons is equal to the number of features of the training data. Next, is the hidden layer where, the number of neuron is user dependent or it is needed to be decided based on some criteria. If fully connected network architecture is considered the total number of connection between input and hidden layer is equal to $N_{i} \times N_{h}$, where $N_{i}$ is the number of neurons in the input layer, $N_{h}$ is the number of neurons in hidden layer. Finally, if the output layer contains $N_{o}$ number of neurons, the total number of connections between hidden and output layer is $N_{i} \times N_{h}$. Thus, total number of neural connections of such network is equal to $N_{h} \times\left(N_{i}+N_{o}\right)$. Hence, the training phase of the NN can be framed as an optimization problem as follows; Minimize $E(\mathbf{W})$, $\mathbf{W}=\left[W_{0}, W_{1}, \ldots W_{N}\right]$. ' $E$ ' denotes the objective (error) function, ' $\mathbf{W}$ ' is an ' $N$ ' dimensional vector where ' $N$ ' is equal to $N_{h} \times\left(N_{i}+N_{o}\right)$. Thus, 'W' represents the weight vector corresponding to a NN. Magnitude of every component of the vector varies in between ' 0 ' and ' 1 '. The metaheuristic algorithm minimizes the objective function and finally finds the optimal weight vector that provides minimum objective function value.

In the current study, a well-known metaheuristic algorithm known as flower pollination algorithm (FPA) [42] has been employed to train the NNs for the hybrid model. The metaheuristic is inspired by the pollination process of the flowers. Biotic and cross pollination have been used for global pollination process while the local search is accomplished by means of abiotic and self-pollination. Natural pollinators are simulated by using levy flights. Studies have revealed the ingenuity of the FPA algorithm over other metaheuristics [2,7,24,40]. Motivated by this, this algorithm has been employed in the current study in training the NNs of the HNN model. The objective function used in the optimization process is root mean squared error (RMSE) [11].

\subsection{Hybrid Neural Network model}

The present work proposes a Hybrid Neural Network (HNN) model which is supported by a multistep training phase. Initially, the data points are grouped into a suitable number of clusters. In the current study, fuzzy c-means algorithm [17] has been used to cluster the data points into a suitable number of clusters. The suitable number of clusters has been decided by trial and error method. Next for each cluster a separate $\mathrm{NN}$ is employed and trained to build the model. It is motivated by the fact that for highly distributed data it could be hard for a single NN. Thus, it could be beneficial, if the data points are clustered into reasonable number of groups and apply a separate NN for each cluster.

Therefore, different NNs will learn the different part of the whole pattern. The hypothesis is tested by comparing this HNN model with two other well-known NN based models. For training the NNs of HNN model, scaled conjugate gradient descent algorithm [32], has been employed, which is benchmarked against backpropagation algorithm. Fig. 1 depicts the HNN model for two clusters. In the present study fuzzy c-means algorithm is employed on initial data points to group tem into a suitable number of clusters. Thereafter, data points of each cluster are used to train one $\mathrm{NN}$ thereby enabling a particular neural network to learn the 
Chatterjee S., Datta B., Dey N.: Hybrid Neural Network based rainfall...

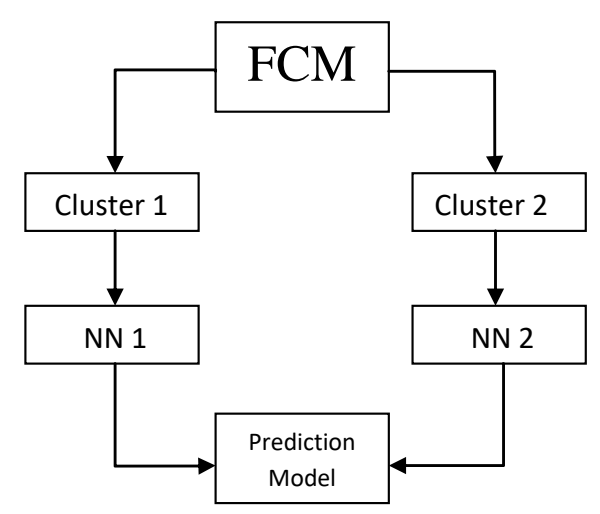

Fig. 1 Hybrid neural network model.

pattern for a specific group of data points of the whole data. During testing phase, membership of the testing sample is determined and the corresponding NN trained for that cluster is used to predict the rainfall value. The number of ANNs employed for every cluster can be increased further to enhance the accuracy. However, this increment might affect the time complexity. Thus, in the current study, only one ANN is employed for every cluster to keep the model simple.

\section{Experimental methodology}

The proposed HNN method has been used to predict the rainfall quantity of the southern part of state West Bengal of India using a data set which is collected from Dumdum meteorological station. The features are tabulated in Tab. I. Fig. 2 depicts the experimental methodology. Before applying the predictive models for the classification task, a preprocessing phase is employed in order to find out the most important features in this classification task. Finding out the important features, help in overcoming the problems with over fitting and computational complexity. There are eight features in the actual dataset. Using the feature

\begin{tabular}{ll}
\hline Attribute & Details \\
\hline Min_Pressure & Minimum pressure (in mb) \\
Min_Vapour & Minimum vapor quantity \\
Min_Relative Humidity & Minimum relative humidity \\
Min_Temperature & Minimum temperature \\
Max_Pressure & Maximum pressure (in mb) \\
Max_Vapour & Maximum vapor quantity \\
Max_Relative Humidity & Maximum relative humidity \\
Max_Temperature & Maximum temperature \\
\hline
\end{tabular}

Tab. I Set of initial features of the dataset. 


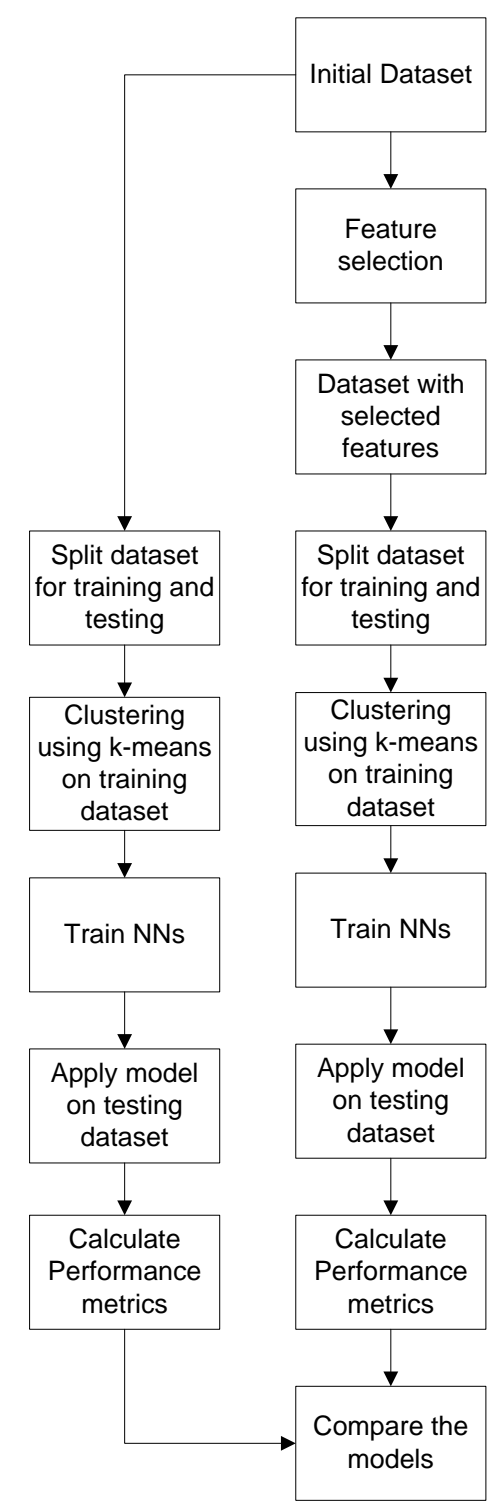

Fig. 2 Figure demonstrates the flow of experiment in the current study.

selection phase we would like to find out the set of most suitable features for rainfall prediction. To accomplish this task, a greedy forward selection algorithm [18] is utilized. The algorithm starts with feature subsets of size one. Then in subsequent iterations the feature subsets of size two, three, and more are evaluated to find out the best feature set.

The greedy forward selection algorithm is computationally efficient and it explicitly works with sparse solutions. In the current study the initial set of features 
are depicted in Tab. I. The feature selection phase is applied on this set of features. Tab. III. depicts the finally selected set of features.

After applying the pre-processing stage, fuzzy c-means algorithm is used to cluster the dataset into a reasonable number of clusters. In the current study the dataset is clustered into two clusters. 10-fold cross validation method is used in testing phase of the proposed model. The experiments are carried out to establish the significance of feature selection phase. Results have been reported for different models before and after feature selection. To establish the ingenuity of the proposed model the proposed HNN model is compared with three different models namely BPNN which is NN trained with simple backpropagation algorithm, secondly it is compared with MLP-FFN which is trained with scaled conjugate gradient descent algorithm. The proposed model is also compared with a similar HNN model with the exception that the NNs are trained with gradient descent algorithm. The comparison is done in term of confusion matrix based performance measuring metrics such as accuracy, precision, recall, and f-measure [27].

\section{Experimental results \& discussion}

The experimental setup described in Section 3 has been followed and the models in the current study are implemented in MATLAB (version 2015a) in a 4GB Intel i3 machine. For implementing NNs for the proposed HNN model Neural Network Toolbox (MATLAB 2015a) has been used. For HNN (gradient descent), scaled conjugate gradient descent algorithm has been used to train the network. Cross entropy was chosen as the objective function. Fuzzy c-means from Fuzzy Logic Toolbox (MATLAB 2015a) has been used for implementing the initial clustering phase. Tab. II reports the features in the initial dataset. It has eight different features namely the maximum and minimum values of pressure, vapor, relative humidity and temperature. After applying the greedy forward selection algorithm the weights assigned to the features by the algorithm are tabulated in Tab. II. A threshold 0.8 of weight value is used to select the final set of features. The threshold value is decided by a trial and error method. The threshold value is varied within range 0.2 to 0.9 and the set of features corresponding to every such threshold value

\begin{tabular}{lc}
\hline Attribute & Assigned Weight \\
\hline Min_Pressure & 1.00 \\
Min_Vapour & 0.00 \\
Min_Relative Humidity & 0.37 \\
Min_Temperature & 0.01 \\
Max_Pressure & 0.90 \\
Max_Vapour & 0.81 \\
Max_Relative Humidity & 0.43 \\
Max_Temperature & 0.37 \\
\hline
\end{tabular}

Tab. II Feature set before feature selection operation. 
is used to test the classifier accuracy. The set of features corresponding to highest accuracy is selected. The features selected are tabulated in Tab. III with their corresponding weights.

\begin{tabular}{lc}
\hline Attribute & Assigned Weight \\
\hline Min_Pressure & 1.00 \\
Max_Pressure & 0.90 \\
Max_Vapour & 0.81 \\
\hline
\end{tabular}

Tab. III Feature set after feature selection.

After applying the fuzzy c-means algorithm the clusters are depicted in Fig. 3. The number of clusters is set to two. It has been decided by a trial and error method. It has been found that if the number of clusters is 2 , the achieved accuracy is best. A threshold on membership value 0.75 is used to get the crisp membership of the data points and the plot is obtained. Thereafter, for each cluster a separate $\mathrm{NN}$ is employed which is trained by FPA algorithm. The experimental setup of FPA algorithm is as in [24]. For rest of the models, the dataset is directly fed to the models and using 10-fold cross validation the performance metrics are tabulated. A plot of mean squared error vs. epoch for the data points in the cluster 1 is shown in Fig. 4. Fig. 5 depicts a plot of epoch wise gradient values for the MLP-FFN classifier. The plot reveals that the gradient is gradually decreasing and is expected

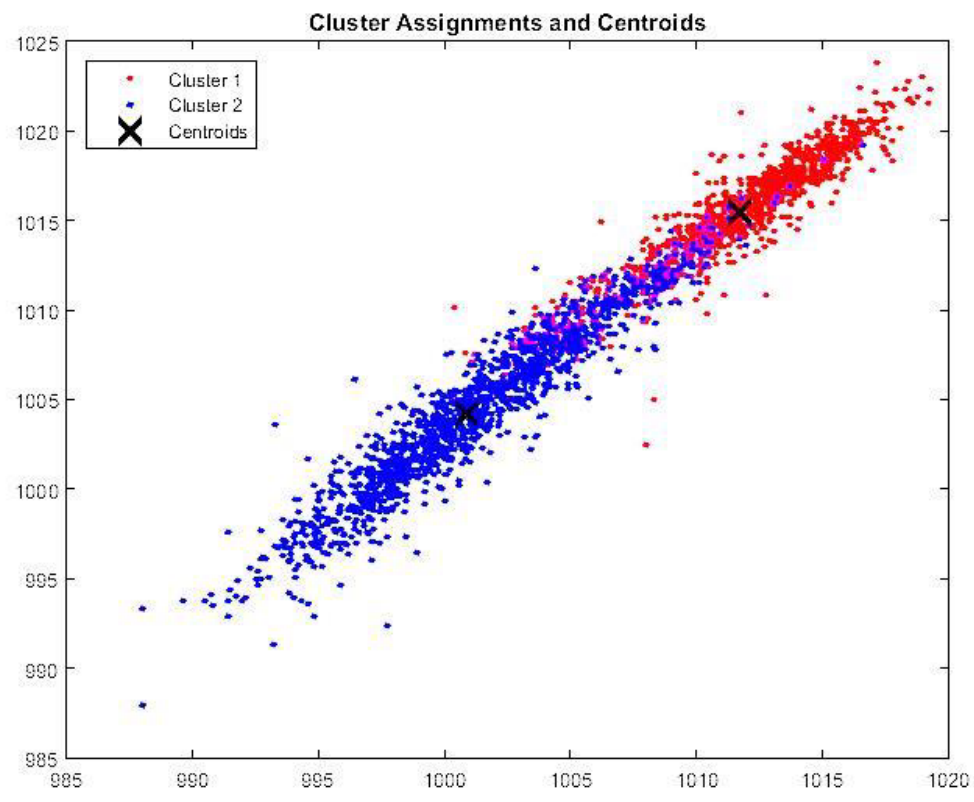

Fig. 3 Plot of data points after being clustered by fuzzy c-means algorithm. Where ' $x$ ' axis depicts Minimum Pressure and ' $y$ ' axis represents Maximum Pressure. 
Chatterjee S., Datta B., Dey N.: Hybrid Neural Network based rainfall...

to converge to global optima. However, the values are fluctuating which indicates that the global optima might not be achieved.

The current work proposed the HNN model for a classification task. The data points are classified into two different classes. One class corresponds to data points

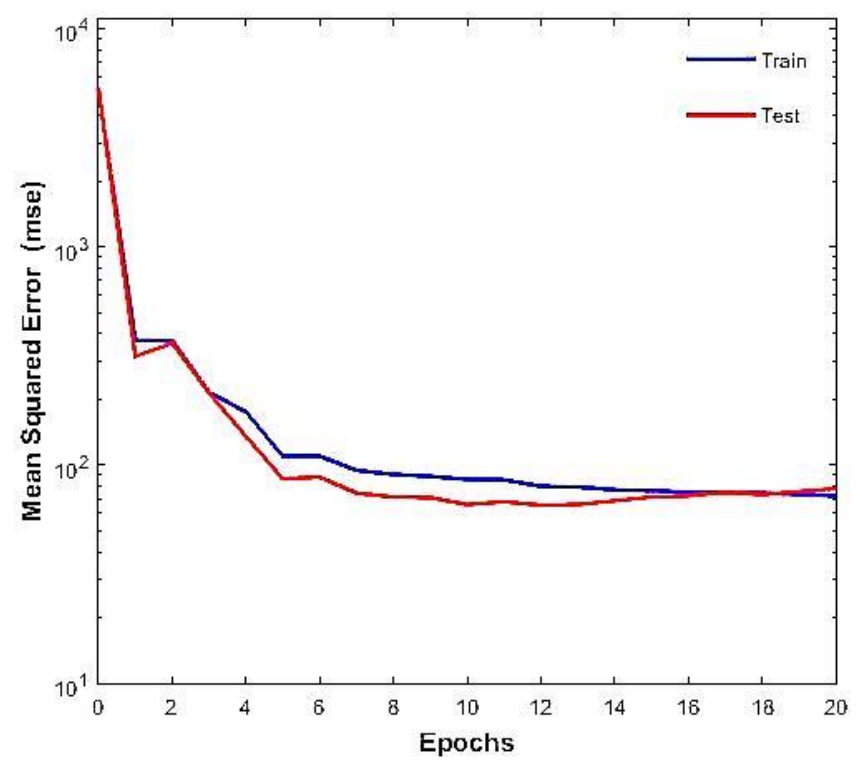

Fig. 4 Plot of Mean squared error vs. epoch for training phase of $N N$ being trained by data points of cluster 1 .

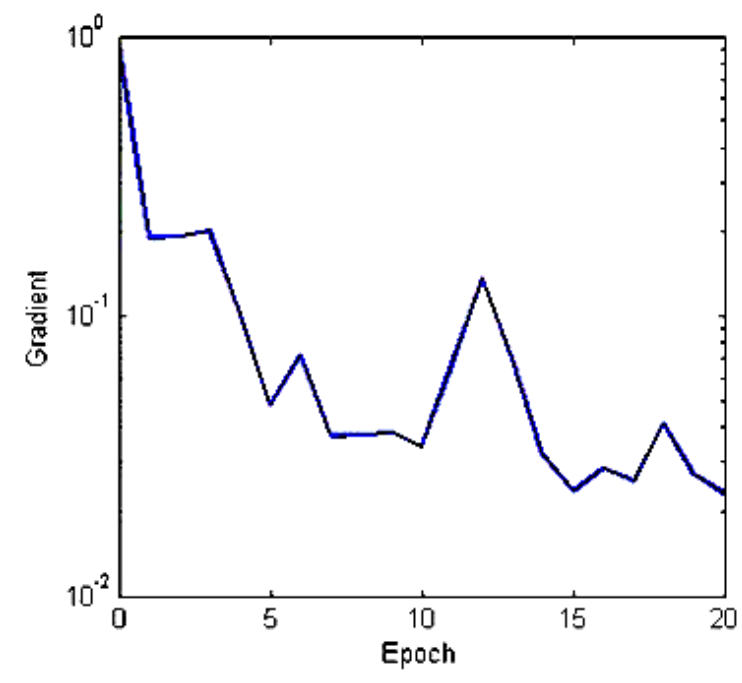

Fig. 5 Plot of gradient vs. epoch for training phase of $N N$ being trained by data points of cluster 1 . 
where rainfall quantity is zero and the second class corresponds to positive rainfall quantity. The experimental results to study the effect of feature selection phase are tabulated in Tab. IV. It reveals that the BPNN method achieved an accuracy of $78.7 \%$ before and $84.7 \%$ after feature selection phase. A similar trend is observed for other performance measures as well. A significant improvement is found in the case of MLP-FFN classifier as well. The performance of proposed HNN trained by FPA algorithm is also improved after applying the feature selection method.

\begin{tabular}{lcccccc}
\hline \multirow{2}{*}{$\begin{array}{l}\text { Performance } \\
\text { Measure }\end{array}$} & $\begin{array}{c}\text { Before } \\
\text { feature } \\
\text { selection }\end{array}$ & $\begin{array}{c}\text { After } \\
\text { feature } \\
\text { selection }\end{array}$ & $\begin{array}{c}\text { Before } \\
\text { feature } \\
\text { selection }\end{array}$ & $\begin{array}{c}\text { After } \\
\text { feature } \\
\text { selection }\end{array}$ & $\begin{array}{c}\text { Before } \\
\text { feature } \\
\text { selection }\end{array}$ & $\begin{array}{c}\text { After } \\
\text { feature } \\
\text { selection }\end{array}$ \\
\hline Accuracy & 78.70 & 84.70 & 82.65 & 88.32 & 84.26 & 89.54 \\
Precision & 77.45 & 83.55 & 78.54 & 84.58 & 88.75 & 92.44 \\
Recall & 68.56 & 71.75 & 76.89 & 81.68 & 86.56 & 90.43 \\
F-Measure & 72.73 & 77.20 & 77.71 & 83.10 & 87.64 & 91.43 \\
\hline
\end{tabular}

Tab. IV Experimental results to study the effect of feature selection.

To establish the ingenuity of the proposed method a comparative study is reported in Tab. V. These results have been obtained after applying feature selection phase. The BPNN model achieved an accuracy of $84.7 \%$, precision of $83.55 \%$, recall of $71.75 \%$, and f-measure of $77.20 \%$. The MLP-FFN model has achieved an accuracy of $88.32 \%$ and $84.58 \%, 81.68 \%$, and $83.1 \%$ of precision, recall and f-measure respectively. This is a significant improvement over BPNN. Next, the HNN, where every NN is trained by gradient descent algorithm, achieved an accuracy of $89.54 \%$ which is significantly better than other models. The same trend can be observed with other performance metrics as well. It indicates that applying the hybrid architecture can improve the performance of classifiers in predicting the rainfall. However, HNN (FPA) achieved an accuracy of $92.52 \%$ which surpassed the performance of all the other classifier. This establishes the ingenuity of the proposed model.

\begin{tabular}{lcccc}
\hline $\begin{array}{l}\text { Performance } \\
\text { Measure }\end{array}$ & BPNN & MLP-FFN & $\begin{array}{c}\text { HNN } \\
\text { (Gradient Descent) }\end{array}$ & $\begin{array}{c}\text { HNN } \\
\text { (FPA) }\end{array}$ \\
\hline Accuracy & 84.70 & 88.32 & 89.54 & 92.52 \\
Precision & 83.55 & 84.58 & 92.44 & 94.28 \\
Recall & 71.75 & 81.68 & 90.43 & 92.48 \\
F-Measure & 77.20 & 83.10 & 91.43 & 93.37 \\
\hline
\end{tabular}

Tab. V Comparative Analysis of the proposed models.

To establish the ingenuity of the proposed HNN model a statistical significance test is carried out. Wilcoxon rank test [41] with $5 \%$ level of significance is carried 
out. The null hypothesis is considered as there is no significant difference between the mean of performance metric values of different groups. On the other hand the alternative hypothesis is that there exists a significant difference. The P-values obtained from HNN vs. MLP-FFN and HNN vs. BPNN are reported in Tab. VI. In all the cases the Null hypothesis is rejected with high confidence. Besides, the $\mathrm{P}$-values indicate that the performance obtained by the proposed model is not random, thereby establishing its ingenuity.

\begin{tabular}{cc}
\hline HNN vs. BPNN & HNN vs. MLP-FFN \\
\hline $2.9 \mathrm{E}-07$ & $2.4 \mathrm{E}-06$ \\
\hline
\end{tabular}

Tab. VI P-values of Wilcoxon Rank test.

\section{Conclusion}

Rainfall is one of the most important factors that affect the weather condition with a greater extent. It is also plays an important role in natural disasters such as flood, drought etc. Agriculture and farming is heavily dependent on the timings of rainfall. Thus, it would be immensely helpful if the rainfall could be predicted accurately in order to get the benefit of the rainfall in the required sectors and at the same time to prevent natural disasters as well. The current study proposed a novel hybrid neural based approach to build a robust and accurate model for rainfall prediction. The proposed model is tested by using a dataset collected by Dumdum meteorological center situated at state West Bengal of eastern part of India. The hybrid model is supported by a feature selection phase. The experimental results have revealed that the feature selection phase can significantly improve classifier performance for rainfall prediction. Besides, the extensive comparative analysis reveals that the proposed HNN model is highly efficient in predicting rainfall. As a future scope of the study (i) further investigation can be done by increasing the number of ANNs per cluster to observe the effect on time complexity, (ii) a comparative study of the proposed model with other classifiers in different domains of science and engineering can be carried out.

\section{References}

[1] AHMAD M., MOURSHED M., REZGUI Y. Trees vs Neurons: Comparison between random forest and ANN for high-resolution prediction of building energy consumption. Energy and Buildings. 2017 Jul 15, 147, 77-89, doi: 10.1016/j.enbuild.2017.04.038.

[2] ALAM D., YOUSRI D., ETEIBA M. Flower pollination algorithm based solar PV parameter estimation. Energy Conversion and Management. 2015 Sep 1, 101, 410-22, doi: 10.1016/j . enconman.2015.05.074.

[3] AWAN S., ASLAM M., KHAN Z., SAEED H. An efficient model based on artificial bee colony optimization algorithm with Neural Networks for electric load forecasting. Neural Computing and Applications. 2014 Dec 1, 25(7-8), 1967-78, doi: 10.1007/s00521-014-1685-y. 


\section{Neural Network World 6/2018, 497-510}

[4] AZAR A., EL-SAID S., BALAS V., OLARIU T. Linguistic hedges fuzzy feature selection for differential diagnosis of Erythemato-Squamous diseases. In Soft computing applications 2013 (pp. 487-500). Springer, Berlin, Heidelberg, doi: 10.1007/978-3-642-33941-7_43

[5] BALL R., TISSOT P., ZIMMER B., STERBA-BOATWRIGHT B. Comparison of random forest, artificial neural network, and multi-linear regression: a water temperature prediction case. In Seventh conference on artificial intelligence and its applications to the environmental sciences 2009 Jan 12 (pp. 1-6), doi: 10.1109/emes.2017.7980368.

[6] BAUGHMAN D., LIU Y. Neural networks in bioprocessing and chemical engineering. Academic press, 2014 Jun 28, URL: https://www.elsevier.com/books/neural-networks-inbioprocessing-and-chemical-engineering/baughman/978-0-12-083030-5.

[7] BINH H., HANH N., DEY N. Improved cuckoo search and chaotic flower pollination optimization algorithm for maximizing area coverage in wireless sensor networks. Neural computing and applications. 2018 Oct 1, 30(7), 2305-17, doi: 10.1007/s00521-016-2823-5.

[8] CAO Z., CHENG L., ZHOU C., GU N., WANG X., TAN M. Spiking neural network-based target tracking control for autonomous mobile robots. Neural Computing and Applications. 2015 Nov 1, 26(8), 1839-47, doi: 10.1007/s00521-015-1848-5.

[9] ChATterJeE S., DATTA B., SEN S., DEY N., DEBNATH N. Rainfall prediction using hybrid neural network approach. In Recent Advances in Signal Processing, Telecommunications 83 Computing (SigTelCom), 2018 2nd International Conference on 2018 Jan 29 (pp. 67-72). IEEE, doi: 10.1109/sigtelcom.2018.8325807.

[10] CHATTERJEe S., GHOSH S., DAWN S., HORE S., DEY N. Forest Type Classification: A hybrid NN-GA model based approach. In Information systems design and intelligent applications 2016 (pp. 227-236). Springer, New Delhi, doi: 10.1007/978-81-322-2757-1_23.

[11] ChatTerJeE S., SARKAR S., HORE S., DEY N., AShOUR A., BALAS V. Particle swarm optimization trained neural network for structural failure prediction of multistoried RC buildings. Neural Computing and Applications. 2017 Aug 1, 28(8), 2005-16, doi: 10. 1007/s00521-016-2190-2.

[12] CiAnCiO C., AMBrogio G., GAGLiARDi F., MUSMANNO R. Heuristic techniques to optimize neural network architecture in manufacturing applications. Neural Computing and Applications. 2016 Oct 1, 27(7), 2001-15, doi: 10.1007/s00521-015-1994-9.

[13] DASH R., PANIGRAHY C., SUBUDHI B. A comparison between RNN and RBF NN techniques for the detection of stator inter-turn fault of an induction motor. In Electrical, Electronics, Signals, Communication and Optimization (EESCO), 2015 International Conference on 2015 Jan 24 (pp. 1-7). IEEE, doi: 10.1109/iecr.2010.5720163.

[14] DEHURI S., CHO S. A hybrid genetic based functional link artificial neural network with a statistical comparison of classifiers over multiple datasets. Neural Computing and Applications. 2010 Mar 1, 19(2), 317-28, doi: 10.1007/s00521-009-0310-y.

[15] DUBEY A. Artificial neural network models for rainfall prediction in Pondicherry. International Journal of Computer Applications. 2015 Jan 1, 120(3), doi: 10.5120/21210-3910.

[16] DUTTA B., RAY A., PAL S., PATRANABIS D. A connectionist model for rainfall prediction. Neural Parallel \& Scientific Comp. 2009 Mar 1, 17(1), 47-58, doi: 10.12732/npsc.

[17] GAO J., WANG J., ZHOU Y. A Novel Fuzzy C-means Clustering Algorithm to Improve the Recognition Accuracy. Science \& Engineering Research Support soCiety, 2015 Oct 24, doi: 10.14257/astl.2015.111.44.

[18] GUYON I., ELISSEEFF A. An introduction to variable and feature selection. Journal of machine learning research. 2003, 3(Mar), 1157-82, doi: 10.1007/978-981-10-4965-1_1

[19] GAO S., NING B., DONG H. Adaptive neural control with intercepted adaptation for timedelay saturated nonlinear systems. Neural Computing and Applications. 2015 Nov 1, 26(8), 1849-57, doi: $10.1007 / \mathrm{s} 00521-015-1855-6$.

[20] GOLASOWSKI M., LITSCHMANNOVÁ M., KUCHAŘ Š., PODHORÁNYI M., MARTINOVIČ J. Uncertainty modelling in Rainfall-Runoff simulations based on parallel Monte Carlo method. Neural Network World. 2015 Jul 14, 25(3), 267-86, doi: 10.14311/nnw. 2015. 25.014 . 
Chatterjee S., Datta B., Dey N.: Hybrid Neural Network based rainfall...

[21] HAN J., PEI J., KAMBER M. Data mining: concepts and techniques. Elsevier, 2011 Jun 9, doi: 10.1016/b978-0-12-381479-1.00001-010.1016/b978-0-12-381479-1.00001-0

[22] HARTIGAN J., WONG M. Algorithm AS 136: A k-means clustering algorithm. Journal of the Royal Statistical Society. Series C (Applied Statistics). 1979 Jan 1, 28(1), 100-8, doi: $10.2307 / 2346830$.

[23] HUNG N., BABEL M., WEESAKUL S., TRIPATHI N. An artificial neural network model for rainfall forecasting in Bangkok, Thailand. Hydrology and Earth System Sciences. 2009 Aug 7, 13(8), 1413-25, doi: 10.5194/hessd-5-183-2008.

[24] JAGATHEESAN K., ANAND B., SAMANTA S., DEY N., SANTHI V., ASHOUR A., BALAS V. Application of flower pollination algorithm in load frequency control of multiarea interconnected power system with nonlinearity. Neural Computing and Applications. 2017 Dec 1, 28(1):475-88, doi: 10.1007/s00521-016-2361-1.

[25] JAIN A. Data clustering: 50 years beyond K-means. Pattern recognition letters. 2010 Jun 1, 31(8), 651-66, doi: 10.1016/j.patrec.2009.09.011.

[26] KAMAL S., DEY N., ASHOUR A., RIPON S., BALAS V., KAYSAR M. FbMapping: An automated system for monitoring Facebook data. Neural Network World. 2017, 27(1), 27, doi: $10.14311 / \mathrm{nnw} .2017 .27 .002$.

[27] KARAYIANNIS N., VENETSANOPOULOS A. Artificial neural networks: learning algorithms, performance evaluation, and applications. Springer Science 83 Business Media, 2013 Jun 29, URL: https://www.springer.com/in/book/9780792392972.

[28] LAZAREVSKA M., KNEZEVIC M., CVETKOVSKA M., TROMBEVA-GAVRILOSKA A Application of artificial neural networks in civil engineering. Tehnički vjesnik. 2014 Dec 21, 21(6), 1353-9, URL: https://hrcak.srce.hr/131329.

[29] MACINTYRE J. Applications of neural computing in the twenty-first century and 21 years of Neural Computing \& Applications. Neural Computing and Applications. 2013 Sep 1, 23(3-4), 657-65, doi: 10.1007/s00521-013-1471-2.

[30] MAREN A., HARSTON C., PAP R. Handbook of neural computing applications. Academic Press, 2014 May 10, URL: https://www.elsevier.com/books/handbook-of-neural-computingapplications/maren/978-0-12-546090-3.

[31] MIRJALILI S., SAREMI S., MIRJALILI S. Designing evolutionary feedforward neural networks using social spider optimization algorithm. Neural Computing and Applications. 2015 Nov 1, 26(8), 1919-28, doi: 10.1007/s00521-015-1847-6.

[32] MØLLER M. A scaled conjugate gradient algorithm for fast supervised learning. Neural networks. 1993 Jan 1, 6(4), 525-33, doi: 10.7146/dpb.v19i339.6570.

[33] NANDA S., TRIPATHY D., NAYAK S., MOHAPATRA S. Prediction of rainfall in India using Artificial Neural Network (ANN) models. International Journal of Intelligent Systems and Applications. 2013 Nov 1, 5(12), 1, doi: 10.5815/ijisa.2013.12.01.

[34] NAYAK D., MAHAPATRA A., MISHRA P. A survey on rainfall prediction using artificial neural network. International Journal of Computer Applications. 2013 Jan 1, 72(16), doi: 10. 5120/12580-9217.

[35] RACZKO E., ZAGAJEWSKI B. Comparison of support vector machine, random forest and neural network classifiers for tree species classification on airborne hyperspectral APEX images. European Journal of Remote Sensing. 2017 Jan 1, 50(1), 144-54, doi: 10.1080/ 22797254.2017 .1299557$.

[36] ROJAS R. Neural networks: a systematic introduction. Springer Science 8 Business Media, 2013 Jun 29, URL: https://www.springer.com/in/book/9783540605058.

[37] SIDDIQUEE M., HOSSAIN M. Development of a sequential Artificial Neural Network for predicting river water levels based on Brahmaputra and Ganges water levels. Neural Computing and Applications. 2015 Nov 1, 26(8), 1979-90, doi: 10.1007/s00521-015-1871-6.

[38] SOCHA K., BLUM C. An ant colony optimization algorithm for continuous optimization: application to feed-forward neural network training. Neural Computing and Applications. 2007 May 1, 16(3), 235-47, doi: 10.1007/s00521-007-0084-z. 


\section{Neural Network World 6/2018, 497-510}

[39] THEUER M., VAVŘÍK R., VONDRÁK V., KUCHǍ̆ Š., ŠÍR B., PORTERO A. Efficient methods of automatic calibration for rainfall-runoff modelling in the Floreon+ system. Neural Network World, 2017, 27(4), pp. 391-414, doi: 10.14311/nnw.2017.27.022.

[40] WANG R., ZHOU Y. Flower pollination algorithm with dimension by dimension improvement. Mathematical Problems in Engineering. 2014, doi: 10.1155/2014/481791.

[41] WILCOXON F., KATTI S.K., WILCOX R. Critical values and probability levels for the Wilcoxon rank sum test and the Wilcoxon signed rank test. Selected tables in mathematical statistics. 1970, 1:171-259, doi: 10.1007/springerreference_205698.

[42] YANG X. Flower pollination algorithm for global optimization. In International conference on unconventional computing and natural computation 2012 Sep 3 (pp. 240-249). Springer, Berlin, Heidelberg, doi: 10.1007/978-3-642-32894-7_27.

[43] ZHANG T. On the consistency of feature selection using greedy least squares regression. Journal of Machine Learning Research. 2009, 10(Mar), 555-68, doi: 10.1109/ijcnn.2015. 7280341 